\title{
La importancia de las fuentes en la selección de artículos de prensa en línea: un estudio de Google Noticias mediante seguimiento ocular (eye-tracking)
}

\author{
Cristòfol Rovira \\ Jofre Capdevila \\ Mari Carmen Marcos *
}

Artículo recibido:

19 de marzo de 2013.

Artículo aceptado:

19 de noviembre de 2013.

\section{RESUMEN}

Se presentan los resultados de un estudio realizado en el buscador Google Noticias para determinar la importancia que le dan los usuarios a los elementos principales de una noticia que aparece en un listado de resultados: titular, fuente y resumen. Mediante la técnica de seguimiento ocular (eye-tracking) se analiza la duración de la mirada de 50 usuarios frente a las páginas de resultados y se determina qué elementos captan más tiempo su atención. Los resultados del estudio indican que se presta más atención a las fuentes que al resumen pero que no hay diferencias significativas entre el tiempo dedicado a la fuente con respecto al título, lo cual indica que en los medios en línea los usuarios también

* Los tres autores pertenecen a la Universidad Pompeu Fabra, Barcelona, España (cristofol.rovira@upf.edu); (jofre.capdevila@gmail.com); (mcarmen.marcos@upt. edu)

INVESTIGACIÓN BIBLIOTECOLÓGICA, Vol. 28, Núm. 63, mayo/agosto, 2014, México, ISSN: 0187-358X. pp. 15-28 
consideran a la fuente como elemento para decidir qué artículos leer.

Palabras clave: Buscadores; Buscadores de noticias; Listados de resultados; Servicios de noticias; Comportamiento de los usuarios; Seguimiento ocular (eye-tracking); Fuentes de información.

\section{Abstract}

The importance of sources in the selection of online newspaper articles: a study of Google News using eye-tracking

Cristòfol Rovira, Jofre Capdevila and Mari-Carmen Marcos

This paper presents the results of a study of the news search engine Google News to determine the degree of importance users lend to headlines, sources and summaries as these appear in the lists of search results. By use of eye-tracking, researchers tallied the duration of the gaze of fifty users viewing search results displays to determine which of these elements captured their attention longest. The results indicate that more attention is paid to sources than to the summaries; while there are no significant differences between time spent viewing sources versus the title, indicating that online media users also consider the sources when deciding which articles to pursue.

Keywords: Search engines; News search engines; Search engines result lists; SERP, news services; User behavior; Eye-tracking, information sources.

\section{INTRODUCCIÓN}

T a prensa en línea ha sido objeto de numerosos estudios a menudo centraLdos en análisis comparativos (Díaz-Noci y Meso Ayerdi, 1998; Schultz, 1999) o en valorar algún aspecto concreto, como el contenido multimedia (Díaz-Noci, 2009; Guallar, Rovira y Ruiz, 2010), las redes sociales (Rodríguez-Martínez, Codina y Pedraza-Jiménez, 2010), la recuperación de información (Martínez Rubio, 2008; Guallar y Abadal, 2009; Rubio Lacoba, 2010) o los contenidos de los blogs (Sánchez Vigil et al., 2010). 
Menos comunes son los estudios de lectura de prensa a través de buscadores especializados como el que aquí abordamos, Google Noticias. Se trata de un servicio de búsqueda de noticias que reúne artículos recogidos de diversas fuentes de prensa, de manera que el usuario pueda encontrar la información sobre un mismo tema procedente de distintos medios.

Los buscadores de prensa, como Google Noticias, presentan tres bloques de información en cada uno de los ítems de los listados de resultados: el título de la noticia, la fuente de donde toman la noticia y un resumen (Ilustración 1). A partir de esos tres bloques informativos, el usuario tiene que decidir si consulta o no la noticia completa. A esta relativa poca información se suma el hecho de que leemos en diagonal. Nicholas Carr (2011), finalista del Premio Pulitzer, señala que Internet fomenta un picoteo rápido y distraído de pequeños fragmentos de información de muchas fuentes a una gran velocidad, sin dejar espacio a la reflexión y al pensamiento crítico.

En un contexto así, la decisión de qué noticia leer termina siendo un proceso rápido guiado por el interés personal por el tema y la información provista por el buscador. En este estudio nos preguntamos hasta qué punto los lectores consideran que la fuente de origen de la noticia es un dato importante para tomar su decisión sobre la continuidad de su lectura. En formato papel parece quedar más claro el rigor o el punto de vista de cada fuente, pero en la Web, y más en un buscador de noticias, ¿qué papel juega la fuente en las decisiones de los lectores?

En este estudio partimos de la hipótesis de que cuando los usuarios utilizan las nuevas tecnologías para informarse en temas de actualidad tienden a ser menos rigurosos en la evaluación de la información. Creemos que la sobrecarga de información lleva a seleccionar las noticias en gran parte condicionados por un titular atractivo. Así pues, partimos de la idea de que cuando un usuario revisa un listado de resultados en un buscador de noticias la mayoría de las miradas se concentrarán en los títulos y por tanto se dedicará mucho más tiempo a mirar los titulares que las fuentes.

Para realizar este estudio se llevó a cabo un test con usuarios en el laboratorio utilizando un dispositivo de seguimiento de la mirada (eye-tracker). Esta tecnología ha sido usada en trabajos anteriores para conocer el comportamiento visual de las personas que revisan páginas de resultados de los buscadores (Granka, Joachims y Gay, 2004; Rele y Duchowski, 2005; González-Caro y Marcos, 2011; Nielsen y Pernice, 2009), pero por ahora no se ha publicado ninguna investigación sobre páginas de resultados de noticias.

La estructura del artículo es la siguiente: en la sección "Trabajos previos" se presentan los estudios que han servido como punto de partida al presente; en la sección "Google Noticias" se detalla el diseño experimental llevado a 
cabo; a continuación se ofrecen los resultados del análisis de los tests y finalmente se presentan las conclusiones de este estudio.

\section{TRABAJOS PREVIOS}

El seguimiento de la mirada (más conocido por su forma en inglés eye-tracking) es una técnica que permite conocer la posición de los ojos y los movimientos de éstos cuando se está realizando una acción determinada, para lo cual utiliza una tecnología de rayos infrarrojos capaz de detectar las pupilas. Esta técnica ha sido utilizada principalmente en investigación sobre el sistema visual, en psicología cognitiva y en el diseño de productos. El sector de mercadotecnia la ha usado para mejorar la colocación de productos en las estanterías de los supermercados, para escoger espacios publicitarios en las páginas web, para aumentar las proporciones (ratios) de conversión de las compras en línea, o para hacer benchmarking (comparar las acciones de los usuarios en su sitio web con las que hacen los de la competencia). En los últimos años esto se ha aplicado de forma bastante habitual en la evaluación de sitios web y especialmente en estudios de usabilidad de interfaces digitales, la mayoría de los cuales han sido motivados por intereses comerciales. El libro de Jakob Nielsen y Kara Pernice (2009) presenta numerosos ejemplos de aplicación del seguimiento ocular en la usabilidad, en sus capítulos se encuentran estudios que han sentado las bases para evaluar menús de navegación, enlaces, imágenes y publicidad en el diseño web.

Uno de los campos de estudio que también ha recibido mucha atención han sido las interfaces de búsqueda y recuperación de información, sobre todo aplicadas a los resultados de los buscadores de Internet, las llamadas SERPS (Search Engine Results Pages o páginas de resultados de los motores de búsqueda). En este ámbito la tecnología de seguimiento ocular ha permitido hacer algunos hallazgos importantes, como por ejemplo la evidencia del llamado golden triangle a partir de los mapas de calor. El "triángulo de oro" (Enquiro, 2003) es aquella zona que capta más miradas de la mayoría de los usuarios, y está situada en la esquina superior izquierda de un listado de resultados.

A estos resultados siguieron otros como el de Granka, Joachims y Gay (2004). En sus tests pusieron de manifiesto que aunque el número de clics sea muy superior en el primer resultado, los usuarios pasan el mismo tiempo analizando el primero que el segundo, y que visualizan hasta la posición 5 y 6 ante preguntas o tareas más complicadas. Más adelante, Guan y Cutrell (2007) observaron que los usuarios clican con mucha más frecuencia los dos 
primeros resultados, aunque la respuesta a su búsqueda no se encuentre en ellos. Un estudio de la Cornell University (Pan et al., 2007) cambia de orden los resultados y concluye que los usuarios tienen "fe ciega" en la clasificación que realizan los buscadores, independientemente de la relevancia de estos resultados.

Otros estudios tienen en cuenta las distintas zonas de la página de resultados (resultados orgánicos y publicidad, o incluso con más detalle: título, snippet y URL de cada resultado). Rele y Duchowski (2005) detectan que el snippet (el resumen) recibe un porcentaje mayor de fijaciones que los títulos, mientras que las URL son las que menos fijaciones reciben. Estas conclusiones quedan reforzadas por estudios más recientes como el de Marcos y González-Caro (2010) y el de González-Caro y Marcos (2011), que concluyen que el elemento más mirado en los resultados es el resumen (44\% del tiempo), seguido del título (39 $\%)$ y la URL (17 \%).

En el área específica de la prensa digital, los estudios más citados realizados con eye-tracking son los cuatro del Poynter Institute (Eyetrack I en 1990-91, Eyetrack II en 1999-2000, Eyetrack III en 2003-2004 y Eyetrack IV en 2006-2007). Entre los resultados se muestran mapas de calor de páginas web de diarios que proporcionan interesantes sugerencias sobre cuándo y cómo incorporar publicidad, elementos multimedia y textos cortos en las portadas de la prensa digital.

El estudio que se plantea en este trabajo no analiza páginas web de prensa digital ni de buscadores generalistas, sino que busca saber qué influencia tiene la fuente de información al momento de escoger una noticia en el agregador de noticias Google Noticias. Por ahora no ha habido trabajos publicados que utilicen tecnología de movimiento de los ojos (eye-tracking) para estudiar esta plataforma.

\section{Google Noticias}

Google Noticias es la edición que hace el mercado español de Google News, un agregador y buscador de noticias que rastrea de forma constante y automática la información de los principales medios de comunicación en línea del ámbito geográfico del usuario. Este servicio, lanzado por Google Inc. en 2002, estuvo en versión de prueba hasta el 2006 y hasta junio de 2012 existían 72 ediciones regionales para sectores geográficos concretos, entre ellos España. El agregador está disponible en 28 lenguas diferentes y funciona a partir de un algoritmo de agregación automático que cada 15 minutos rastrea los más de 25.000 medios digitales de los cuales nutre la base de datos 
y vuelca las noticias de los últimos 30 días en su servidor. En este trabajo vamos a estudiar la interfaz de la página de resultados, la cual describimos a continuación.

En cada ítem del listado de resultados de Google Noticias hay hasta nueve elementos de información diferentes (datos de enero de 2012). Hay cuatro elementos básicos que aparecen siempre en todos los resultados y que proporcionan la información principal de la noticia (Ilustración 1):

1. Título. El titular principal de la noticia; es un enlace activo que permite ir al artículo original.

2. Fuente principal. El nombre de la fuente/medio de comunicación que ha publicado la noticia.

3. Fecha. La fecha de publicación de la noticia.

4. Resumen. Es la captura de los 200 primeros caracteres del artículo original.

Luego hay tres elementos que aparecen sólo cuando Google encuentra noticias muy similares a la principal en otros medios.

5. Títulos secundarios. Títulos clicables de una selección de noticias similares al titular principal.

6. Fuentes secundarias. Fuentes o medios de información donde se han publicado las noticias listadas como secundarias.

7. Más información. Acceso al listado completo de noticias similares a la principal.

Finalmente, suele aparecer una imagen relacionada con la noticia, aunque no en todos los casos.

8. Imagen vinculada con la noticia.

9. La fuente de donde ha sido extraída la imagen.

Así pues, lo más usual es que aparezcan elementos de los tres grupos.

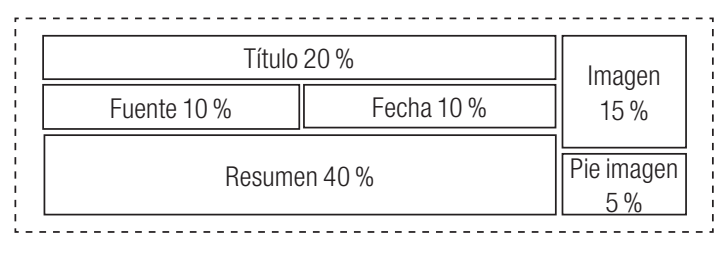

Ilustración 1. Áreas de un ítem del listado de resultados de Google News. 


\section{Metodología}

\section{Participantes}

La selección de los usuarios que realizarán el test es un proceso complejo si se intentan cumplir los requisitos para poder generalizar los resultados de la muestra a una población determinada. Normalmente los estudios de usabilidad con seguimiento ocular son de tipo más cualitativo y no buscan una generalización, sino sólo detectar anomalías que hay que mejorar en el diseño de algún elemento de una página web, y por tanto las muestras de usuarios suelen ser pequeñas, entre 10 y 15 participantes. En cambio, esta investigación tiene una orientación más cuantitativa porque se pretenden identificar evidencias sólidas sobre el grado de influencia de las fuentes en el momento de decidir qué ítem clicar en la página de resultados de Google Noticias. Por tanto, se ha sido más exigente en la selección de la muestra de individuos, a pesar de las limitaciones inherentes de todo proceso de reclutamiento, especialmente con presupuestos ajustados.

Dadas las características del test se decidió que nos interesaban individuos que fueran usuarios o usuarios potenciales del servicio de Google Noticias, y por tanto se estableció que el perfil para realizar el test fuera de usuarios habituales de Internet y mayores de edad.

En general se aconseja que el número de usuarios para un estudio cuantitativo tiene que ser superior a 30 participantes (Marcos, Nettleton y Sáez, 2012). De acuerdo con las previsiones de tiempos, medios y recursos para desarrollar el proyecto, se decidió que participarían 60 usuarios en el estudio. Posteriormente se descartaron los usuarios en los que el eye-tracker había grabado menos del $80 \%$ de sus fijaciones, así como dos usuarios con datos incoherentes o excesivamente apartados de las medias. La muestra definitiva contó con 50 individuos distribuidos de la forma siguiente:

Tabla 1. Distribución de los usuarios del experimento.

\begin{tabular}{|c|c|c|c|c|c|c|}
\hline Edades & Hombres & $\%$ & Mujeres & $\%$ & Total & $\%$ \\
\hline 18 - 20 años & 5 & $10 \%$ & 14 & $28 \%$ & 19 & $38 \%$ \\
\hline 21 - 25 años & 0 & $0 \%$ & 7 & $7 \%$ & 7 & $14 \%$ \\
\hline 26 - 30 años & 4 & $8 \%$ & 2 & $4 \%$ & 6 & $12 \%$ \\
\hline 31 - 35 años & 3 & $6 \%$ & 3 & $6 \%$ & 6 & $12 \%$ \\
\hline 36 - 40 años & 3 & $6 \%$ & 2 & $4 \%$ & 5 & $10 \%$ \\
\hline $41-50$ años & 3 & $6 \%$ & 2 & $4 \%$ & 5 & $10 \%$ \\
\hline+50 años & 1 & $2 \%$ & 1 & $2 \%$ & 2 & $4 \%$ \\
\hline Total & 19 & $38 \%$ & 31 & $62 \%$ & 50 & $100 \%$ \\
\hline
\end{tabular}




\section{Dispositivo del eye-tracking}

Los tests se realizaron con un dispositivo de eye-tracker modelo Tobii 1750. Se trata de un monitor que integra un sistema de luz infrarroja que capta el movimiento ocular. Esta tecnología detecta con gran precisión la posición de la mirada en la pantalla, así como el tiempo de permanencia de la mirada en un punto concreto del monitor. Estos datos objetivos precisan de una interpretación a nivel cognitivo (Hassan y Herrero, 2007), y tradicionalmente ésta se ha hecho asumiendo que cuando la persona fija la mirada en un elemento está pensando en ese elemento. A pesar de que esta relación no es siempre cierta, es lo suficientemente consistente como para obtener resultados sólidos, especialmente si el número de usuarios que participan en el estudio es relativamente alto.

\section{Diseño del experimento}

El objetivo de este estudio es conocer el comportamiento visual del usuario ante una búsqueda de información. Por lo tanto, el núcleo del test de usuario se basará en la visualización de los listados de resultados de este servicio.

Para que el estudio fuera sistemático y comparable entre los diferentes usuarios, el test se realizó en iguales condiciones para cada uno intentando homogeneizar al máximo posible la búsqueda inicial para conseguir resultados comparables. Por este motivo se les mostraron a los usuarios unos listados de resultados preelaborados y se les pidió que seleccionaran la noticia más interesante para solucionar una necesidad de información previamente anunciada.

Por otro lado, entendemos que los usuarios podrían estar condicionados por un interés (o desinterés) individual en una temática concreta. Para evitar en lo posible este tipo de sesgo, se elaboraron dos listados de resultados con dos temáticas de actualidad diferentes, uno para cada tarea (Ilustración 2):

Tarea 1: noticia de deportes

Tarea 2: noticia de política/sociedad

Las páginas de resultados mostradas eran capturas de pantalla estáticas tomadas de Google Noticias. Esas capturas fueron ligeramente manipuladas para establecer un pequeño espacio entre las zonas de cada resultado: su título, el resumen, la fuente y la URL. De esa forma sería posible que el eye-tracker tuviera mayor certeza de dónde tenía el usuario exactamente puesta su mirada. 

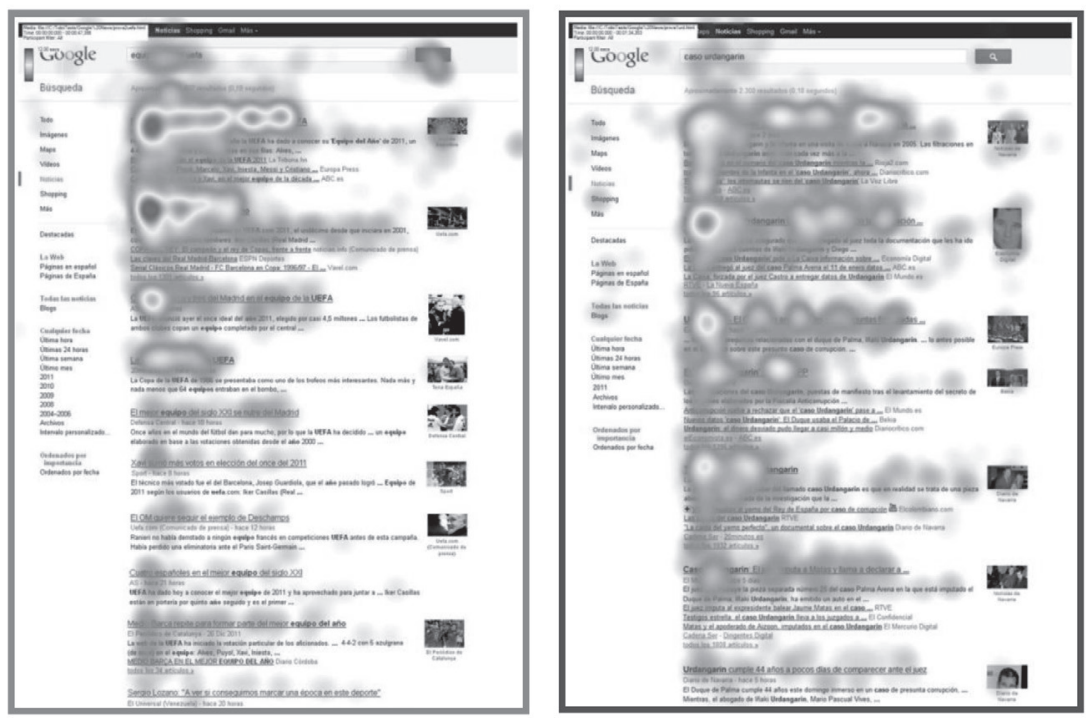

Ilustración 2. Mapas de calor de las listas de resultados analizadas: a la izquierda están las noticias sobre deportes y a la derecha las de política/sociedad.

\section{Métricas}

Como decíamos, cada resultado de Google Noticias tiene una estructura similar formada por áreas relativas al título, la fuente, la fecha, el resumen y la imagen. Cada una de estas áreas de interés (a partir de ahora AOI, Area of Interest) fue delimitada con el software Tobii Studio para obtener métricas para cada una de ellas.

Una de las decisiones importantes en cualquier estudio con eye-tracker es decidir qué métricas se usarán para alcanzar los objetivos planteados. En el contexto de los estudios de usabilidad (Marcos, 2011) se suelen usar métricas relacionadas con el nivel de efectividad y eficiencia de las tareas a realizar, por ejemplo:

- El porcentaje de usuarios que han fijado su mirada en una AOI.

- El tiempo transcurrido desde el inicio de la tarea hasta finalizarla.

- El número de fijaciones que los usuarios hacen en cada AOI.

- Tiempo de duración de las fijaciones en cada AOI.

En este estudio se usó el tiempo de duración de las fijaciones como métrica principal para determinar la importancia que los usuarios le otorgan a cada zona de cada ítem de resultados. 


\section{Resultados}

Debido a los objetivos del estudio, el análisis de datos estará centrado en el elemento "Fuente principal", que será comparado con las principales áreas que aparecen en todos los ítems (el título y el resumen). Los datos han sido normalizados dado que todas las AOI tienen tamaños distintos. La normalización se ha realizado dividiendo el tiempo de fijación de la mirada en cada AOI por la superficie de estas AOI, y se ha obtenido un dato normalizado de tiempo por unidad de superficie.

Tabla 2. Tiempo normalizado de duración de las fijaciones en las áreas principales.

\begin{tabular}{|l|c|c|c|c|c|}
\hline & Título & Resumen & Fuente & $\begin{array}{c}\text { Diferencias } \\
\text { Título y } \\
\text { Fuente }\end{array}$ & $\begin{array}{c}\text { Diferencias } \\
\text { Resumen y Fuente }\end{array}$ \\
\hline Usuarios & 50 & 50 & 50 & 50 & 50 \\
\hline Media & 787,660 & 232,080 & 983,520 & $-195,860$ & $-751,440$ \\
\hline Shapiro-Wilk & 0,884 & 0,731 & 0,809 & 0,909 & 0,774 \\
\hline Shapiro-Wilk Sig. P-valor & 0,000 & 0,000 & 0,000 & 0,001 & 0,000 \\
\hline
\end{tabular}

Tabla 3. Prueba de los rangos con signo de Wilcoxon.

\begin{tabular}{|l|c|c|}
\hline & Título y Fuente & Resumen y Fuente \\
\hline$Z$ & $-1,057$ & $-5,913$ \\
\hline Sig. P-valor & 0,290 & 0,000 \\
\hline
\end{tabular}

En el estudio estadístico estamos trabajando con datos numéricos con muestras relacionadas, el mismo individuo observa todas las AOI analizadas. Nos interesa especialmente identificar si hay diferencias significativas entre la variable "Título" en relación a la "Fuente" y de la variable "Resumen" también en relación a la "Fuente". De acuerdo con la prueba de Shapiro-Wilk ninguna de las variables sigue la distribución normal, como podemos ver en las dos columnas de las diferencias (Tabla 2), donde el p-valor es menor a 0,05 . Por tanto, no se pueden aplicar pruebas paramétricas, como la T de Student, para saber si hay diferencias significativas. Al ser datos dependientes se ha aplicado la prueba no paramétrica de los rangos con signo de Wilcoxon y hemos constatado que sólo hay diferencias estadísticamente significativas entre las variables "Resumen" y "Fuente" ya que obtenemos un valor de p-valor inferior a 0,05 . En cambio, no hay diferencias significativas entre las variables "Título" y "Fuente" donde se obtiene un p-valor de 0,290 (Tabla 3). Aunque el centro de atención de esta investigación está en el comportamiento de los 
usuarios ante las zonas donde aparecen las fuentes de información, también cabe señalar que hay diferencias significativas entre el título y el resumen y que éstas son a favor del título.

Las diferencias en datos de tiempo normalizado entre fuente y resumen son importantes. Vemos que hay una diferencia de 751 a favor de la fuente, o sea que hay un aumento de la atención de un $323 \%$ al pasar del resumen a las fuentes, o dicho de otra forma, la fuente recibe 4,24 veces más atención que el resumen (Tabla 2).

Por tanto, de acuerdo con los datos obtenidos y respetando la significación estadística, podemos afirmar que el área de la fuente recibe una mayor atención que el resumen.

\section{Conclusiones}

En este estudio se ha analizado el comportamiento de un grupo de usuarios ante los listados de resultados de Google Noticias en el momento de tomar la decisión sobre qué ítem clicar. Nuestro propósito era conocer la importancia que le dan los usuarios a las fuentes de las noticias que aparecen en la descripción de cada ítem del listado de resultados. Para ello se ha usado un sistema de seguimiento ocular que ha registrado el tiempo de fijación de la mirada de los usuarios en las distintas zonas de los listados de resultados. Para evitar el sesgo que pudiera generarse en las áreas de mayor superficie se han usado datos relativos del tiempo de fijación por unidad de superficie.

De acuerdo con los datos obtenidos, los usuarios prestan más atención a las fuentes que al resumen. En cambio, no hay diferencias significativas entre el tiempo en que se observan los títulos en relación con el tiempo dedicado a mirar las fuentes, tal como planteábamos en la hipótesis. Esto indica que los usuarios se fijan en la fuente de la que procede la información tanto como en el título, lo cual no corrobora la hipótesis inicial que afirmaba que los medios digitales hacen que las personas sean menos rigurosas al escoger un artículo, al menos así se desprende de este estudio para el caso de Google Noticias.

Aunque pensamos que estos indicios son sólidos, para generalizar las conclusiones a la población general sería necesario realizar estudios complementarios que los confirmaran. Por otra parte, también habría que hacer nuevas investigaciones para confirmar que el valor alto de tiempo relativo de fijaciones de la mirada en el área de las fuentes en relación con el resumen es debido al interés por este contenido y no una consecuencia de que esta área se sitúe en la parte superior de cada ítem, justo debajo del título. 
Periodísticamente hablando, el estudio aporta una nueva visión sobre cómo se enfrentan los usuarios a los servicios de noticias y pretende extraer conclusiones sobre la importancia que tienen las cabeceras de los medios en el momento de decidir qué ítem se clicará del listado de resultados de un buscador de noticias.

Nota:

Este trabajo forma parte del proyecto de investigación CSO2011-22691Nuevas estrategias de publicidad y promoción de las marcas turísticas españolas en la Web. Ministerio de Ciencia e Innovación, 2012-2014.

\section{BibliografíA}

Carr, Nicholas (2011), Superficiales. ¿Qué está haciendo internet con nuestras mentes?, Madrid: Taurus.

Díaz-Noci, Javier (2009), "Multimedia y modalidades de lectura: una aproximación al estado de la cuestión", en Comunicar, núm. 33, pp. 213-219.

_- Meso Ayerdi, Koldo (1998), "Desarrollo del periodismo electrónico", en El Profesional de la Información, vol. 7, núm. 12, pp. 411.

Enquiro/Eyetools Google Report (2003), artículo disponible en: http: //eyetools.com/research_google_eyetracking_heatmap.html

González-Caro, Cristina; Marcos, Mari-Carmen (2011), "Different Users and Intents: An Eye-tracking Analysis of Web Search", en Irwin King, Wolfgang Nejdl, Hang Li (eds.), wsdm'11 Fourth acm International Conference on Web Search and Data Mining (Kowloon, Hong Kong, February 09-12, 2011), New York: ACM.

Granka, Laura; Joachims, Thorsten; Gay, Geri (2004), "Eye-tracking analysis of user behavior in WWW search", en Proceedings of the 27th Annual international acm sigir Conference on Research and Development in information Retrieval (Sheffield, United Kingdom, July 25-29, 2004), New York: ACM Press, pp. 478-479, disponible en: http://www.cs.cornell.edu/People/tj/publications/gran ka_etal_04a.pdf

Guallar, Javier; Abadal, Ernest (2009), "Evaluación de hemerotecas de prensa digital: indicadores y ejemplos de buenas prácticas", en El Profesional de la Información, noviembre-diciembre, vol. 18, núm. 3, pp. 255-269. 
_- Rovira, Cristòfol; Ruiz, Sara (2010), "La multimedialidad en la prensa digital. Presencia de elementos multimedia y sistemas de recuperación en los principales diarios digitales españoles", en El Profesional de la Información, noviembre-diciembre, vol. 19, núm. 6, pp. 620-629.

Guan, Zhiwei; Cutrell, Edward (2007), "An eye tracking study of the effect of target rank on Web search", en Procs of the Sigchi conf on buman factors in computing systems, New York: ACM Press, disponible en: http://dl.acm.org/citation.cfm?id=1240691

Hassan Montero, Yusef; Herrero Solana, Víctor (2007), "Eye-Tracking en Interacción Persona-Ordenador", en No sólo usabilidad, octubre, disponible en: http://www.nosolousabilidad.com/articulos/ eye-tracking.htm

Marcos, Mari-Carmen (2011), Disseny centrat en l'usuari: com millorar l'experiència d'ús en llocs web, Barcelona: COBDC.

Marcos, Mari-Carmen; González-Caro, Cristina (2010), "Comportamiento de los usuarios en la página de resultados de los buscadores. Un estudio basado en eye tracking”, en El Profesional de la Información, julio-agosto, vol. 19, núm. 4, pp. 348-358.

—- Nettleton, David; Sáez, Diego (2012), "Evaluation of User Search Behavior using a Web Search Log and Eye Tracking data", en Proceedings of the BCS HCI. People E Computers XXVI (Birmingham, UK), pp. 262-267.

Martínez Rubio, Rosa (2008), "La recuperación de la información en los periódicos digitales valencianos”, en Guillermo López García, Comunicación local y nuevos formatos periodísticos en internet: cibermedios, confidenciales y weblogs, Valencia: Servei de Publicacions de la Universitat de València.

Nielsen, Jakob; Pernice, Kara (2009), Eyetracking web usability, Berkeley: New Riders Press.

Pan, B. et al. (2007), "In Google we trust: Users' decisions on rank, position, and relevance", en Journal of Computer-Mediated Communication, 12 (3), article 3, disponible en: http://jcmc.indiana. edu/vol12/issue3/pan.html

Poynter Institute (2004), Eyetrack III: online news consumer bebavior in age of multimedia, disponible en: http://www.poynter.org/extra/ eyetrack2004/index.htm

Rele, Rachana S.; Duchowski, Andrew T. (2005), "Using eye tracking to evaluate alternative search results interfaces", en Proceedings of the Human Factors and Ergonomics Society (Sept 26-30, Orlando, FL, HFES), disponible en: http://andrewd.ces.clemson.edu/research/ vislab/docs/Final_HFES_Search.pdf

Rodríguez-Martínez, Ruth; Codina, Lluís; Pedraza-Jiménez, Rafael (2010), "Cibermedios y web 2.0: modelo de análisis y resultados de aplicación”, en El Profesional de la Información, enero-febrero, vol. 9, núm. 1, pp. 35-44. 
Rubio Lacoba, María (2010), "Mejor que un buscador, un 'encontrador' ", en Documentación de las ciencias de la información, núm. 33, pp. 273-287.

Sánchez Vigil, Juan Miguel; Marcos Recio, Juan Carlos; Olivera Zaldua, María (2010), "Presencia y contenidos de los blogs en los principales diarios españoles", en El Profesional de la Información, noviembre-diciembre, vol. 19, núm. 6, pp. 602-609.

Schultz, Tanjev (1999), "Interactive Options in Online Journalism: A Content Analysis of 100 U.S. Newspapers", en Journal of Computer -Mediated Communication, vol. 5, issue 1, September. 